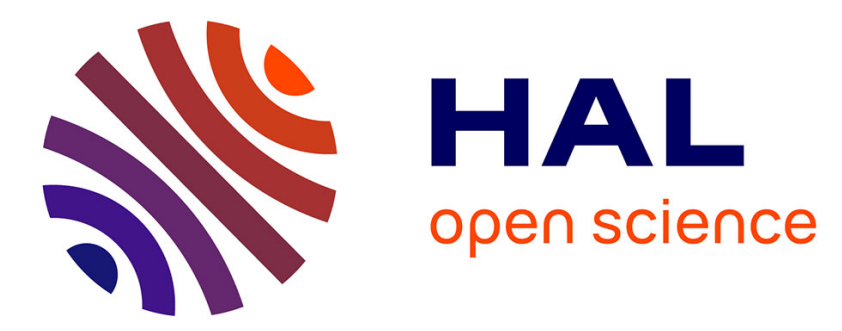

\title{
Détermination des coefficients d'élasticité et de rupture d'âmes nids d'abeilles Nomex $(R)$ : homogénéisation périodique et simulation numérique
}

Laurent Gornet, Gilles Marckmann, Marc Lombard

\section{- To cite this version:}

Laurent Gornet, Gilles Marckmann, Marc Lombard. Détermination des coefficients d'élasticité et de rupture d'âmes nids d'abeilles Nomex $(\mathrm{R})$ : homogénéisation périodique et simulation numérique. Mechanics \& Industry, 2005, 6 (6), pp.595-604. 10.1051/meca:2006005 . hal-01006727

\section{HAL Id: hal-01006727 \\ https://hal.science/hal-01006727}

Submitted on 24 Oct 2016

HAL is a multi-disciplinary open access archive for the deposit and dissemination of scientific research documents, whether they are published or not. The documents may come from teaching and research institutions in France or abroad, or from public or private research centers.
L'archive ouverte pluridisciplinaire HAL, est destinée au dépôt et à la diffusion de documents scientifiques de niveau recherche, publiés ou non, émanant des établissements d'enseignement et de recherche français ou étrangers, des laboratoires publics ou privés. 


\title{
Détermination des coefficients d'élasticité et de rupture d'âmes nids d'abeilles Nomex ${ }^{\circledR}$ : homogénéisation périodique et simulation numérique
}

\author{
Laurent Gornet $^{1, a}$, Gilles Marckmann ${ }^{1}$ et Marc Lombard ${ }^{2}$ \\ 1 École Centrale de Nantes, Institut de recherche en Génie Civil et Mécanique, GeM, UMR CNRS 6183 , 1 rue de la Noë, \\ BP 92101, 44321 Nantes Cedex 3, France \\ 2 Marc Lombard Architecture Navale, Le Sextant, rue de la Trinquette, 17000 La Rochelle, France
}

\begin{abstract}
Résumé - Le présent travail concerne la détermination des propriétés mécaniques des âmes nids d'abeilles de forme hexagonale et de leurs versions sur-expansées rectangulaires. Ces âmes en papiers Nomex ${ }^{\circledR}$ sont présentes sur les voiliers multicoques de courses océaniques entièrement réalisés à partir de sandwichs à peaux de tissus carbones. Les caractéristiques mécaniques élastiques de ces âmes sont déterminées par modélisation tridimensionnelle à partir de la théorie de l'homogénéisation des milieux périodiques mise en œuvre dans le cadre de la méthode des éléments finis. L'étude confirme que les symétries des Volumes Élémentaires Représentatifs (VER) conduisent à des propriétés mécaniques homogénéisées orthotropes. Enfin, l'étude des modes de flambement des VERs permet de déterminer les contraintes ultimes de rupture du matériau homogène équivalent. Les techniques d'homogénéisation périodique développées dans cette étude ont été implantées dans le code de calcul éléments finis Cast3M-CEA. Les propriétés mécaniques et les limites à rupture des âmes en nids d'abeilles sont utilisées pour l'étude du renforcement des structures sandwichs proposées sur les trimarans océaniques de 60 pieds.
\end{abstract}

Mots clés : Structures composites / nids d'abeilles / âmes Nomex / éléments finis / rupture / homogénéisation périodique

\begin{abstract}
Failure and elastic properties of Nomex ${ }^{\circledR}$ honeycombs: periodic homogeneization and mechanical simulation. The present study concern the determination of material properties of the hexagonal and rectangular over-expanded honeycombs. These cores made of Nomex ${ }^{\circledR}$ paper are extensively used in the sandwich structures with carbon skins of oceanic multihull sailing race boats. The elastic mechanical properties are determined by a three-dimensional model that involves periodic homogenization techniques in the finite element context. The study confirms that the Representative Volume Element (RVE) symmetries lead to orthotropic homogenized mechanical properties. Finally, RVE buckling modes conduct to determine the ultimate stress of the homogenized material. Periodic homogenized techniques developed in this study are implemented in the finite element code Cast3M-CEA. The honeycomb mechanical properties and ultimate stresses are used to model reinforcements that we proposed for 60's foot Oceanic Trimaran structures.
\end{abstract}

Key words: Composite structures / honeycombs / Nomex cores / finite element method / fracture / periodic homogenization

\section{Introduction}

Cette étude vise à maîtriser la modélisation et la simulation éléments finis du comportement mécanique des âmes nids d'abeilles utilisées dans la réalisation des voiliers océaniques multicoques de type trimarans.

\footnotetext{
a Auteur correspondant : Laurent.gornet@ec-nantes.fr
}

Ces voiliers de course de 60 pieds sont des structures complexes dont la conception repose sur une technologie de pointe issue de l'aéronautique. Ces trimarans sont réalisés à partir de formes gauches en sandwichs carbone époxy. La vérification et l'optimisation de l'échantillonnage du voilier est réalisée à l'aide de simulations éléments finis menées en étroite collaboration avec 


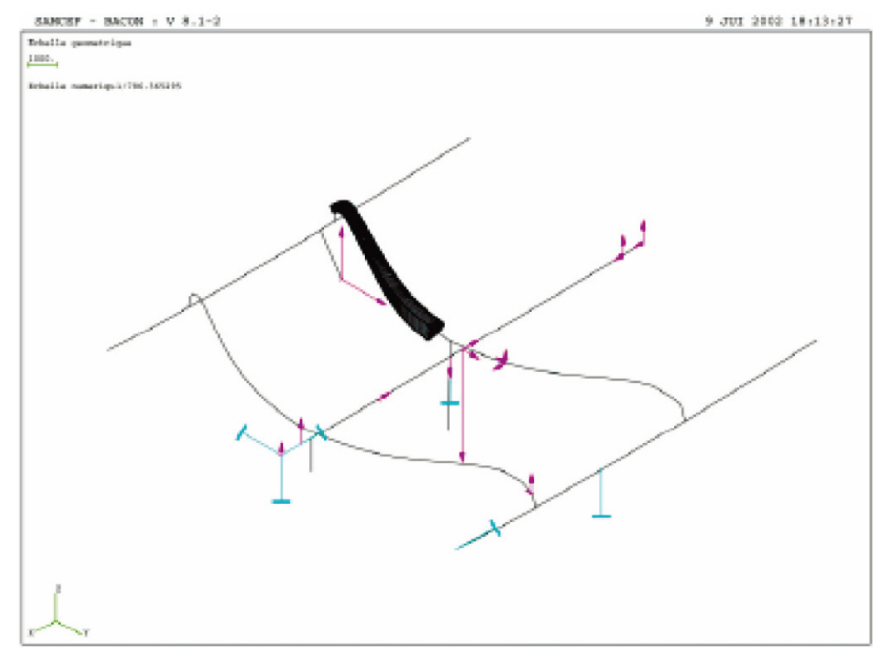

Fig. 1. Maillage mixte poutre/coque d'un trimaran océanique de 60 pieds - plans Lombard.

l'ensemble des acteurs industriels et sportifs du trimaran considéré (Fig. 1) [1]. L'âme de ces sandwichs est généralement constituée de matériaux nids d'abeilles en papier Nomex ${ }^{\circledR}$. Le niveau des compétitions impose un allégement des structures afin de conserver un bon rendement entre le poids et la puissance vélique de ces Formules 1 des Mers. Lors des grandes courses océaniques, comme la Route du Rhum, les conditions atmosphériques extrêmes et le haut niveau des compétitions entraînent parfois un grand nombre d'abandons sur avaries structurelles $[2,3]$. Les âmes en nids d'abeilles constituant les bordés et les carénages des voiliers sont parfois incriminés pour leur manque de durabilité.

Dans la présente étude, la simulation par éléments finis des propriétés mécaniques élastiques homogénéisées d'âmes nids d'abeilles en papier Nomex ${ }^{\circledR}$ est mené à bien. Les contraintes ultimes de ces âmes sont également déterminées. Les formes classiques hexagonales ainsi que leurs versions rectangulaires sur-expansée appelées OX (over extended) sont plus particulièrement étudiées. L'étude repose sur l'utilisation de la théorie de l'homogénéisation des milieux périodiques [4-6] et sur la détermination des modes de flambements de ces structures à parois fortement élancées. Le Volume élémentaire représentatif de chaque type de nids d'abeilles est mis en œuvre dans le code éléments finis Cast3M-CEA [7] afin de déterminer ces propriétés mécaniques homogénéisées. Toute la procédure d'homogénéisation est écrite en langage Gibiane propre à ce code. Afin de montrer la pertinence de l'approche développée dans cette étude, les contraintes ultimes de cisaillement déterminées à partir d'une étude de flambement sont comparées aux valeurs expérimentales issues des travaux du fabricant EuroComposite [8].

\section{Homogénéisation des milieux périodiques}

Lors des simulations éléments finis de validation de solution de conception ou de réparation de voiliers, l'ingénieur est souvent confronté à des matériaux ou à des structures fortement hétérogènes. Toutes les hétérogénéités ne peuvent pas être considérées individuellement et une procédure d'homogénéisation doit alors être menée afin de déterminer les caractéristiques mécaniques équivalentes [4-6].

Les nids d'abeilles constituant l'âme des panneaux sandwichs sont des structures hétérogènes périodiques qui continuent de faire l'objet de nombreuses études [9-15]. Les premiers travaux de Kelsey et al. sur la modélisation des âmes ont porté sur la détermination des caractéristiques mécaniques des modules de cisaillement hors plan [9]. Ces propriétés mécaniques sont déterminantes dans le comportement d'une structure sandwich. La caractérisation du comportement plan pour des formes hexagonales a ensuite été menée par Gibson et Ashby [10-12] afin d'affiner les modèles analytiques. Enfin des approches éléments finis ont récemment été menées par Grediac afin de déterminer les bornes des modules de cisaillement hors plans d'âmes hexagonales [13]. Récemment, des techniques d'homogénéisation associées à des hypothèses cinématiques conduisant à des bornes de Voigt ont été mises en œuvre par Hohe et Becker $[14,15]$ sur des âmes alvéolaires. Notre étude, fondée sur la théorie de l'homogénéisation des milieux périodiques, est menée dans le cadre des éléments finis. Les 21 coefficients du milieu homogénéisé anisotrope tridimensionnel sont déterminés à partir de la géométrie de l'âme et de ses propriétés mécaniques élémentaires. Notre approche est illustrée par des simulations sur des âmes de forme hexagonale classique ainsi que sur leurs versions rectangulaires sur-expansées. Lors de la phase de conception ou de réparation des voiliers océaniques, les propriétés mécaniques des âmes sont une des données essentielles pour le calcul de l'intégrité du voilier. La caractérisation mécanique des peaux de carbone utilisées sur les sandwichs doit également être validée afin de révéler les éventuelles faiblesses en compression ou cisaillement [16]. La mise en œuvre de cette théorie au sein du code de calculs éléments finis Cast3M développé par le CEA [7] a conduit à la réalisation du programme spécifique NidaCore dédié à la caractérisation mécanique des âmes périodiques.

\subsection{Volume élémentaire représentatif}

Les volumes élémentaires représentatifs retenus pour l'homogénéisation des nids d'abeilles à section hexagonale et rectangulaire sur-expansé sont représentés sur la figure 2. Tout volume plus grand peut se déduire par des translations successives du VER. Il convient de noter cependant que le mode de dégradation peut dans certain cas être défini par un VER de dimension supérieure. En effet, les longueurs caractéristiques associées aux mécanismes de déformation et dégradation dans le cas de comportements non-linéaires peuvent parfois englober plusieurs cellules de VER. Dans le cas général d'un matériau de constitution irrégulière, le VER devra inclure 


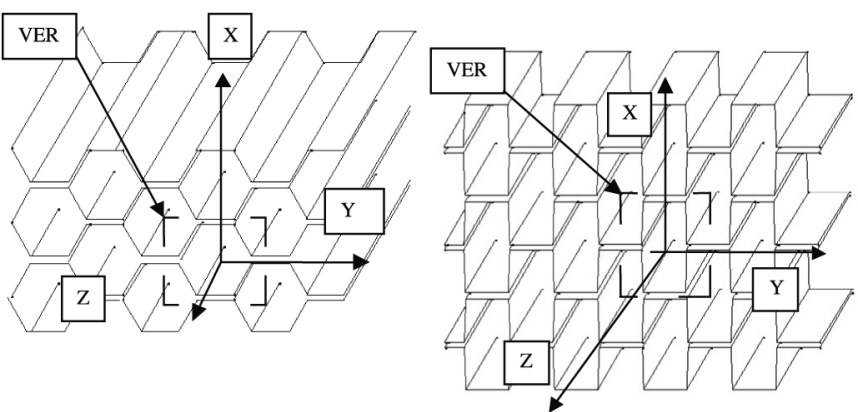

Fig. 2. Cellules de nids d'abeilles hexagonales et sur-expansés.

un grand nombre d'hétérogénéités afin d'être effectivement représentatif du milieu considéré. Dans le cas des deux VER représentés sur la figure 2 , la base $\vec{x}, \vec{y}, \vec{z}$ correspond à la base d'orthotropie du matériau homogénéisé.

\subsection{Méthode des modules effectifs}

La méthode d'homogénéisation utilisée se fonde sur la donnée du tenseur de déformation moyenne $E$ et conduit à déterminer l'opérateur de complaisance $C^{\mathrm{VER}}$ du VER à partir de conditions limites en déplacements imposés sur son bord $\partial Y=\partial Y_{21} \cup \partial Y_{22}$. Le processus d'homogénéisation retenu est basé sur la résolution de 6 problèmes d'élasticité linéaire élémentaires (1-4). Ces six problèmes d'élasticité linéaire à résoudre correspondent à des conditions limites telles que $E_{k h}=1$ et $E_{i j}=0$ si $(i, j) \neq(k, h)$. La détermination de la rigidité de l'âme nécessite quant à elle 21 combinaisons linéaires des six problèmes élémentaires. Les problèmes élémentaires à résoudre sont présentés ci-après :

$$
\begin{aligned}
\operatorname{div}(\sigma(y)) & =0 \quad \sigma(y)=C(y) \varepsilon(u(y)) \quad \text { dans } \quad Y \\
\varepsilon(u) & =\operatorname{grad}_{\mathrm{s}}(u(y)) \\
\sigma(y) \vec{n}_{\text {ext }} & =\overrightarrow{0} \quad \text { sur } \quad \partial Y_{21} \\
\vec{u}(y) & =E \vec{y}+\vec{v}_{\text {per }} \quad \text { sur } \partial Y_{22}
\end{aligned}
$$

Le champ de déplacement $\vec{v}_{\text {per }}$ est périodique et prend naturellement des valeurs égales sur les faces opposées du VER. On utilise le théorème de Hill-Mandel et la notation vectorielle pour calculer les 21 composantes de la rigidité homogénéisée équivalente $C^{\mathrm{VER}}$. Le théorème impose que l'énergie du VER est la moyenne de l'énergie de ces constituants. La loi de comportement inverse du matériau homogène équivalent est déterminée à partir de la relation $S^{\mathrm{VER}}=C^{\mathrm{VER}^{-1}}$.

\section{Aspects logiciel}

La mise en œuvre dans le code éléments finis Cast3M de la méthode d'homogénéisation précédente est réalisée à l'aide du langage de commandes Gibiane [7]. Le centre du repère est au centre du VER et les conditions de périodicité sont appliquées aux faces en vis-à-vis à l'aide

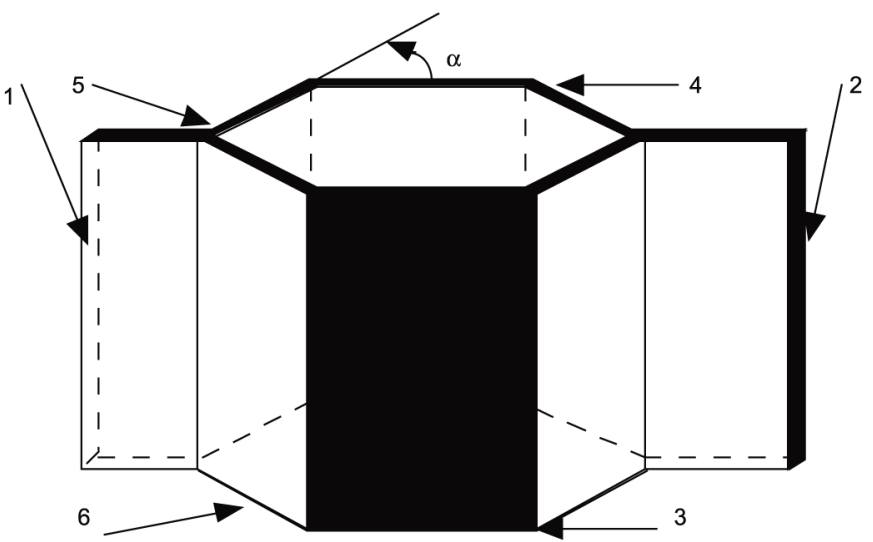

Fig. 3. Cellules de nids d'abeilles hexagonales.

de relations linéaires. Les bords $\partial Y_{22}$ sont les bords 1,2 , $3,4,5$ et 6 définis sur la figure 3 . Le cas particulier des cellules sur-expansées correspond à un angle $\alpha$ de $90^{\circ}$.

\subsection{Conditions de périodicité}

Les conditions de périodicité sont appliquées sur les faces $1,2,3$ et 4 . Les faces inférieure 6 et supérieure 5 sont libres de condition de périodicité. Ces conditions sont prises en compte entre les faces $S_{i}$ et $S_{j}$ en vis-à-vis de la manière suivante : le champ périodique $\vec{v}_{\text {per }}$ a une valeur identique pour deux points $i$ et $j$ en vis-à-vis. En conséquence, le champ périodique est éliminé en combinant les déplacements imposés sur les deux faces :

$$
\begin{array}{r}
\vec{u}_{i}=E_{k l} \vec{y}_{i}+\vec{v}_{\text {per } i} \quad \text { et } \quad \vec{u}_{j}=E_{k l} \vec{y}_{j}+\vec{v}_{\text {per } j} \\
\text { or } \quad \vec{v}_{\text {per } i}=\vec{v}_{\text {per } j}
\end{array}
$$

Par soustraction de ces deux équations, on obtient les relations linéaires qui traduise la périodicité à savoir :

$$
\vec{u}_{i}-\vec{u}_{j}=E_{k l}\left(\vec{y}_{i}-\vec{y}_{j}\right)
$$

\subsection{Calcul de la rigidité et de la souplesse}

Les coefficients du matériau homogénéisé sont déterminés à partir du théorème de Hill-Mandel en écrivant l'énergie à partir des expressions sous forme matricielle. Dans le cas des matériaux anisotropes, on utilise classiquement une notation vectorielle pour représenter leur comportement. La notation retenue dans cette étude diffère des notations classiques car elle est symétrisée et permet de simplifier les expressions des composantes de la rigidité et de la souplesse lors des opérations de changements de base [17]. La géométrie des âmes considérées dans cette étude comporte trois plans de symétrie perpendiculaires ce qui conduit naturellement à un comportement homogénéisé orthotrope. Le comportement est défini par la base d'orthotropie $\vec{x}, \vec{y}, \vec{z}$ (Fig. 2) et les 9 coefficients d'élasticité indépendants dans cette base. 
La condition de Hill-Mandel est écrite sous forme matricielle afin de simplifier la mise en œuvre éléments finis (5) :

$$
\hat{\epsilon}_{I}^{\mathrm{T}} \hat{C}_{I J}^{\mathrm{VER}} \hat{\epsilon}_{J}=\frac{1}{|Y|^{\mathrm{VER}}} \int_{Y} \operatorname{Tr}[\sigma \epsilon] \mathrm{d} Y
$$

Les 21 combinaisons linéaires des six problèmes d'élasticité élémentaires posés sur le VER permettent de déterminer entièrement la matrice de rigidité $\hat{C}_{I J}$. Les équations sont développées ci-après. Il n'y a pas sommation sur les indices dans les formules suivantes :

$$
\begin{gathered}
\epsilon_{I} \hat{C}_{I I}^{\mathrm{VER}} \epsilon_{I}=\frac{1}{|Y|^{\mathrm{VER}}} \int_{Y} \operatorname{Tr}[\sigma \epsilon] \mathrm{d} Y \quad \text { pour } I \in[1,3] \quad(6) \\
\sqrt{2} \epsilon_{I} \hat{C}_{I I}^{\mathrm{VER}} \sqrt{2} \epsilon_{I}=\frac{1}{|Y|^{\mathrm{VER}}} \int_{Y} \operatorname{Tr}[\sigma \epsilon] \mathrm{d} Y \quad \text { pour } I \in[4,6] \\
\epsilon_{I} C_{I I}^{\mathrm{VER}} \epsilon_{I}+2 \epsilon_{I} C_{I J}^{\mathrm{VER}} \epsilon_{J}+\epsilon_{J} C_{J J}^{\mathrm{VER}} \epsilon_{J}= \\
\frac{1}{|Y|^{\mathrm{VER}}} \int_{Y} \operatorname{Tr}[\sigma \epsilon] \mathrm{d} Y
\end{gathered}
$$

pour $(I, J) \in[1,3]^{2}$, avec $(I \neq J)$

$$
\begin{array}{r}
\epsilon_{I} C_{I I}^{\mathrm{VER}} \epsilon_{I}+2 \epsilon_{I} C_{I J}^{\mathrm{VER}} \sqrt{2} \epsilon_{J}+\sqrt{2} \epsilon_{J} C_{J J}^{\mathrm{VER}} \sqrt{2} \epsilon_{J}= \\
\frac{1}{|Y|^{\mathrm{VER}}} \int_{Y} \operatorname{Tr}[\sigma \epsilon] \mathrm{d} Y
\end{array}
$$

$\operatorname{pour}(I, J) \in[1,3] \times[4,6]$

$$
\begin{array}{r}
\sqrt{2} \epsilon_{I} C_{I I}^{\mathrm{VER}} \sqrt{2} \epsilon_{I}+2 \sqrt{2} \epsilon_{I} C_{I J}^{\mathrm{VER}} \sqrt{2} \epsilon_{J}+\sqrt{2} \epsilon_{J} C_{J J}^{\mathrm{VER}} \sqrt{2} \epsilon_{J}= \\
\frac{1}{|Y|^{\mathrm{VER}}} \int_{Y} \operatorname{Tr}[\sigma \epsilon] \mathrm{d} Y
\end{array}
$$

pour $(I, J) \in[4,6]^{2}$, avec $(I \neq J)$.

Les équations (6)-(10) permettent de déterminer facilement la représentation du tenseur des rigidités $\hat{C}_{I J}$ dans la base retenue. Les modules d'Young, coefficient de Poisson ou de couplage sont définis classiquement à partir de la souplesse $\hat{S}_{I J}=\hat{C}_{I J}^{-1}$. Un des avantages de l'approche vectorielle symétrisée introduite dans cette étude est d'obtenir des relations de changement base analytiques identiques sur la rigidité et la souplesse contrairement à la notation classiquement admise [18].

\section{Propriétés mécaniques des nids d'abeilles}

Les nids d'abeilles réalisés en papier Nomex ${ }^{\circledR}$ sont disponibles chez les fabricants pour différentes densités. Dans cette étude, les caractéristiques mécaniques du papier Nomex ${ }^{\circledR}$ sont déterminées de manière inverse par recalage des modules de cisaillement hors plan de l'âme homogénéisée. Les exemples qui illustrent cette étude sont

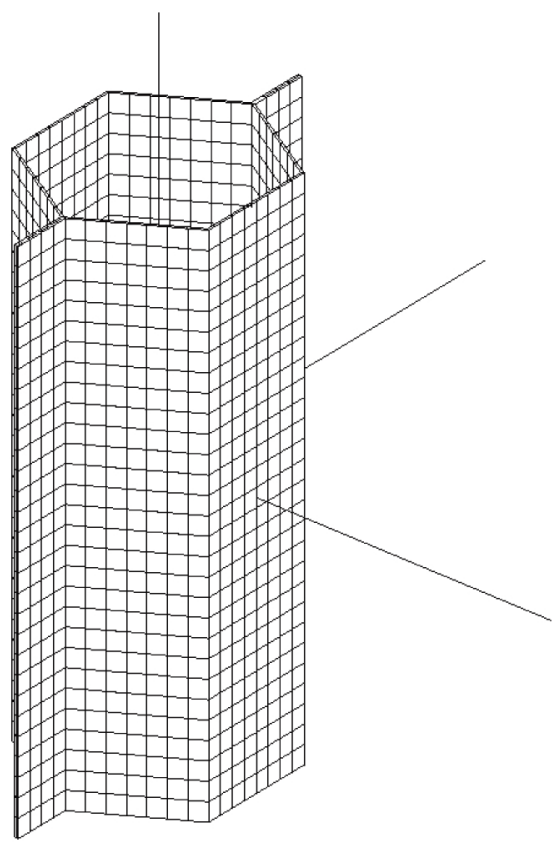

Fig. 4. Maillage d'un VER de nids d'abeilles hexagonales.

déterminés à partir du catalogue « Euro-Composite » [8]. Il convient de noter qu'un produit peut avoir pour une même dimension géométrique, différentes densités, car la quantité de résine déposée peut varier afin de créer une âme plus résistante.

Les propriétés mécaniques en cisaillement hors plan sont déterminées à partir de l'essai rail défini par les normes MIL-C-7438 et MIL-C-8073. Le comportement en compression hors plan est quant à lui déterminé à partir de l'essai DIN-53291. Les caractéristiques mécaniques sont définies à partir d'essais réalisés pour des hauteurs de nids d'abeilles de $12 \mathrm{~mm}$. Les catalogues des fabricants donnent uniquement les propriétés mécaniques les plus importantes vis-à-vis du dimensionnement. Ainsi, les modules de cisaillement hors plan $G_{13}$ et $G_{23}$ et les contraintes de cisaillement de rupture associées $\sigma_{13}$ et $\sigma_{23}$ ainsi que la contrainte d'écrasement $\sigma_{33}$ sont les seules données fournies classiquement par les constructeurs. Ces seules données sont évidemment insuffisantes pour réaliser des études éléments finis tridimensionnelles. L'homogénéisation des milieux périodiques décrite cidessus permet alors de déterminer l'ensemble des caractéristiques mécaniques équivalentes d'un matériau nids d'abeilles.

\subsection{Simulations éléments finis}

Les simulations des propriétés mécaniques homogénéisées sont déterminées à partir des VER des nids d'abeilles présentés sur les figures 4 et 5 . Ils possèdent des hauteurs de $12 \mathrm{~mm}$ conformément au matériau utilisé par le fabriquant pour réaliser les tests mécaniques. Le modèle éléments finis d'un VER comporte 41556 nœuds, soit 6156 éléments hexaèdres à 20 nœuds isoparamétriques 


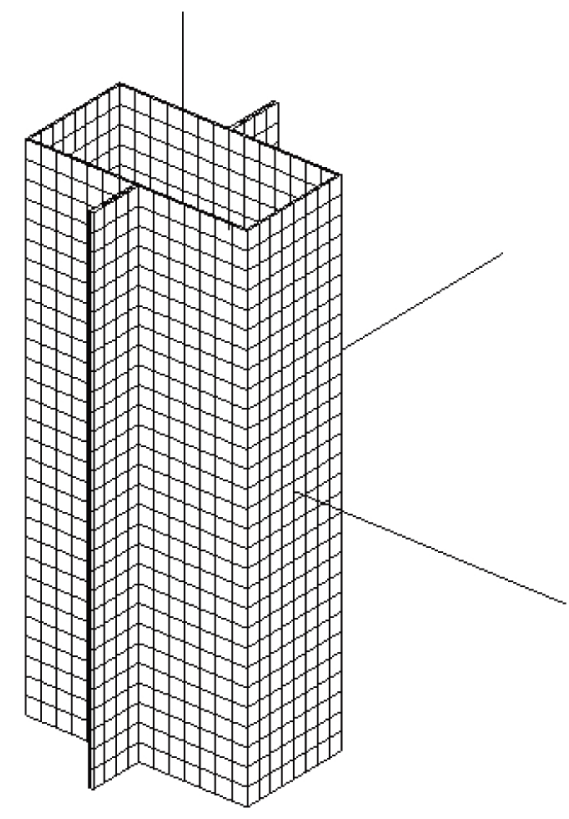

Fig. 5. Maillage d'un VER de nids d'abeilles sur expansés.

à interpolation quadratique de la famille de Serendip. L'intégration numérique est réalisée à l'aide de 27 points de Gauss. Le maillage d'un VER comporte un seul élément dans l'épaisseur élémentaire de la paroi et deux dans les parties à épaisseur double (Figs. 4 et 5). Des études de convergence ont été réalisées pour différentes discrétisations des VER et les maillages retenus sont optimaux vis-à-vis du temps CPU et de la convergence EF. Les simulations éléments finis conduisant aux propriétés mécaniques homogénéisées sont effectuées sous l'hypothèse des petits déplacements et des petites déformations. L'observation des modes de rupture des nids d'abeilles $[10,12,19]$ nous conduit à déterminer les contraintes de rupture de ces matériaux en utilisant la théorie du flambement d'Euler disponible dans le code éléments finis Cast3M. Le langage de programmation de ce code nous permet d'appeler au sein de nos propres développements le module de flambement qui correspond à la recherche de valeurs propres et de vecteurs propres [20]. Les déformations homogénéisées critiques déterminées par le flambement sont introduites dans la loi de comportement homogénéisée afin de déterminer les contraintes ultimes.

\subsection{Caractéristiques mécaniques}

Le calibrage des caractéristiques mécaniques du VER représentant le nid d'abeilles retenu est réalisé en supposant que les parois sont élastiques isotropes. En conséquence, le module d'Young du Nomex ${ }^{\circledR}$ et le coefficient de Poisson sont déterminés afin d'obtenir les modules de cisaillement hors plan du matériau orthotrope équivalent en adéquation avec le catalogue du fournisseur [8].
Tableau 1. Propriétés homogénéisées minimum (Min) et typiques (Typ) des nids d'abeilles ECA-R 4,8 de densité 29, 48 et $64 \mathrm{~kg} \cdot \mathrm{m}^{-3}$ avec une épaisseur de clinquant de (51) $\mu \mathrm{m}$.

\begin{tabular}{ccccccc}
\hline ECA-R & Min & Typ & Min & Typ & Min & Typ \\
MPa & $4,8-29$ & $4,8-29$ & $4,8-48$ & $4,8-48$ & $4,8-64$ & $4,8-64-$ \\
& $(51)$ & $(51)$ & $(51)$ & $(51)$ & $(51)$ & $(51)$ \\
\hline$v_{12}$ & 0,2606 & \multicolumn{2}{c}{0,2606} & 0,2606 \\
$v_{13}$ & 0,2240 & 0,2240 & 0,2240 \\
$v_{23}$ & 0,0219 & \multicolumn{2}{c}{0,0219} & 0,0219 \\
\hline$E_{1}$ & 44,5 & 72,5 & 101,1 & 128,7 & 129,6 & 152,0 \\
$E_{2}$ & 4,4 & 7,1 & 9,9 & 12,6 & 12,7 & 14,9 \\
$E_{3}$ & 79,4 & 129,5 & 180,5 & 229,8 & 231,4 & 271,5 \\
\hline$G_{12}$ & 0,5 & 0,9 & 1,2 & 1,6 & 1,6 & 1,9 \\
$G_{13} \mathrm{~W}$ & 14,6 & 23,9 & 33,3 & 42,4 & 42,7 & 50,1 \\
$G_{23} \mathrm{~L}$ & 8,6 & 14,1 & 19,6 & 25,0 & 25,1 & 29,5 \\
\hline
\end{tabular}

Il convient de noter que ces modules sont déterminés expérimentalement à partir d'essais réalisés sur des éprouvettes de cisaillement. La démarche d'étalonnage du modèle éléments finis est nécessaire pour chaque densité d'âme pour une même géométrie. Le catalogue précise pour chaque géométrie les valeurs minimales (Min) et typiques (Typ) obtenues pendant les essais. En conséquence, deux calibrages sont effectués afin de déterminer les caractéristiques homogénéisées nominales et minimales.

Il convient de noter que les 21 coefficients du milieu homogène équivalent sont calculés. Dans le cas des nids d'abeilles à cellules hexagonales ou rectangulaires, les symétries conduisent à un comportement mécanique homogénéisé orthotrope. Le modèle éléments finis du VER fournit après simulations une approximation de qualité des propriétés mécaniques de l'ensemble du matériau équivalent.

La dénomination ECA-R 4,8-29 (51) représente une âme rectangulaire de cercle inscrit de $4,8 \mathrm{~mm}$ de densité de $29 \mathrm{~kg} . \mathrm{m}^{-3}$ et d'une épaisseur de clinquant de $51 \mu \mathrm{m}$. La dénomination ECA 4,8-29 (51) est équivalente pour une âme de section classique hexagonale. Les propriétés mécaniques homogénéisées des nids d'abeilles de géométrie rectangulaire sont présentées dans les tableaux 1 à 4 et les géométries hexagonales sont fournies dans les tableaux 5 à 8 . Celles de cisaillement hors plan sont présentées sur les figures 6 et 7 pour les matériaux ECA 4,8 et ECA-R 4,8. L'évolution de ces propriétés en fonction de la masse volumique est présentée sur la figure 8 pour le matériau hexagonal et sur la figure 9 pour sa version sur-expansée.

Il convient de noter que les caractéristiques mécaniques du matériau équivalent dépendent dans la réalité de la rigidité des peaux. Ce point peut être abordé afin d'affiner les résultats de cette étude. En effet, les échantillonnages des voiliers de compétition sont déterminés avec des marges de sécurité extrêmement faibles afin d'alléger au maximum la structure. En conséquence les peaux des sandwichs ont généralement des épaisseurs très faibles devant l'épaisseur de l'âme et les phénomènes de flambement restent un facteur de 
Tableau 2. Comparaisons entre les caractéristiques éléments finis (EF) et constructeur (EC). Modules de cisaillement minimum (Min) et typiques (Typ) des nids d'abeilles ECA-R 4,8 de densité 29, 48 et $64 \mathrm{~kg} . \mathrm{m}^{-3}$.

\begin{tabular}{cccccccccccccc}
\hline \multirow{2}{*}{ ECA -R } & \multicolumn{3}{c}{$4,8-29(51)$} & \multicolumn{4}{c}{$4,8-48(51)$} & \multicolumn{4}{c}{$4,8-64(51)$} \\
& \multicolumn{2}{c}{ Min } & \multicolumn{2}{c}{ Typ } & \multicolumn{2}{c}{ Min } & \multicolumn{2}{c}{ Typ } & \multicolumn{2}{c}{ Min } & \multicolumn{2}{c}{ Typ } \\
\hline MPa & EF & EC & EF & EC & EF & EC & EF & EC & EF & EC & EF & EC \\
\hline$G_{13}$ W & 14,6 & 14 & 23,9 & 24 & 33,3 & 36 & 42,4 & 44 & 42,7 & 48 & 50,1 & 56 \\
$G_{23}$ L & 8,6 & 9 & 14,1 & 14 & 19,6 & 18 & 25,0 & 24 & 25,1 & 22 & 29,5 & 26 \\
\hline
\end{tabular}

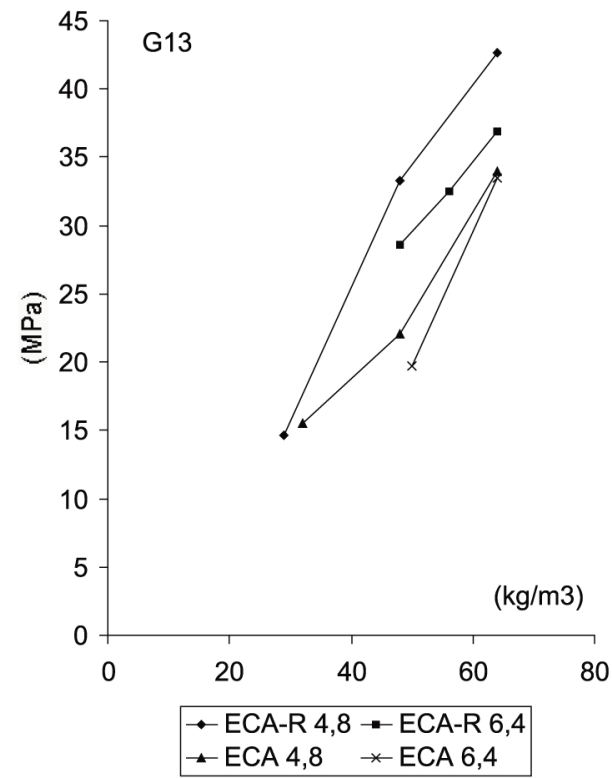

Fig. 6. Évolution du module de cisaillement $G_{13}$ pour deux matériaux à cellules hexagonale et sur-expansée.

Tableau 3. Propriétés homogénéisées minimum (Min) et typiques (Typ) des nids d'abeilles ECA-R 6,4 de densité 48,56 et $64 \mathrm{~kg} \cdot \mathrm{m}^{-3}$ avec une épaisseur de clinquant de (51) $\mu \mathrm{m}$.

\begin{tabular}{ccccccc}
\hline ECA-R & Min & Typ & Min & Typ & Min & Typ \\
MPa & $6,4-48$ & $6,4-48$ & $6,4-56$ & $6,4-56$ & $6,4-64$ & $6,4-64$ \\
& $(51)$ & $(51)$ & $(51)$ & $(51)$ & $(51)$ & $(51)$ \\
\hline$v_{12}$ & 0,2630 & \multicolumn{2}{c}{0,2630} & 0,2630 \\
$v_{13}$ & 0,2236 & \multicolumn{2}{c}{0,2236} & 0,2236 \\
$v_{23}$ & 0,0293 & \multicolumn{2}{c}{0,0293} & 0,0293 \\
\hline$E_{1}$ & 87,2 & 118,2 & 99,2 & 129,2 & 112,7 & 149,4 \\
$E_{2}$ & 11,4 & 15,5 & 13,0 & 16,9 & 14,8 & 19,6 \\
$E_{3}$ & 156,0 & 211,4 & 177,4 & 231,1 & 201,5 & 267,2 \\
\hline$G_{12}$ & 1,4 & 1,9 & 1,6 & 2,1 & 1,8 & 2,4 \\
$G_{13} \mathrm{~W}$ & 28,6 & 38,7 & 32,5 & 42,3 & 36,9 & 49,0 \\
$G_{23} \mathrm{~L}$ & 17,7 & 24,0 & 20,2 & 26,3 & 22,9 & 30,4 \\
\hline
\end{tabular}

risque très important. Dans cette étude, les peaux ne sont pas modélisées et les conditions limites appliquées au VER sont équivalentes à des peaux infiniment rigides.

\subsection{Contraintes à rupture}

Expérimentalement, on constate que le flambement correspond à un accroissement très significatif de l'endommagement des âmes nids d'abeilles en Nomex ${ }^{\circledR}$. Lors

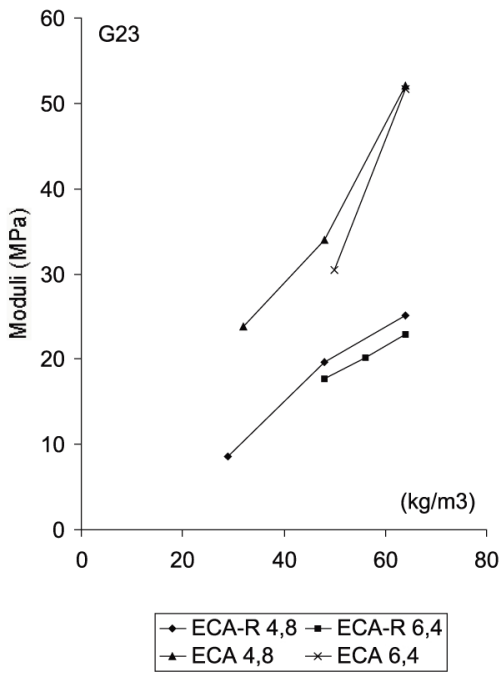

Fig. 7. Évolution du module de cisaillement $G_{23}$ pour deux matériaux à cellules hexagonale et sur-expansée.

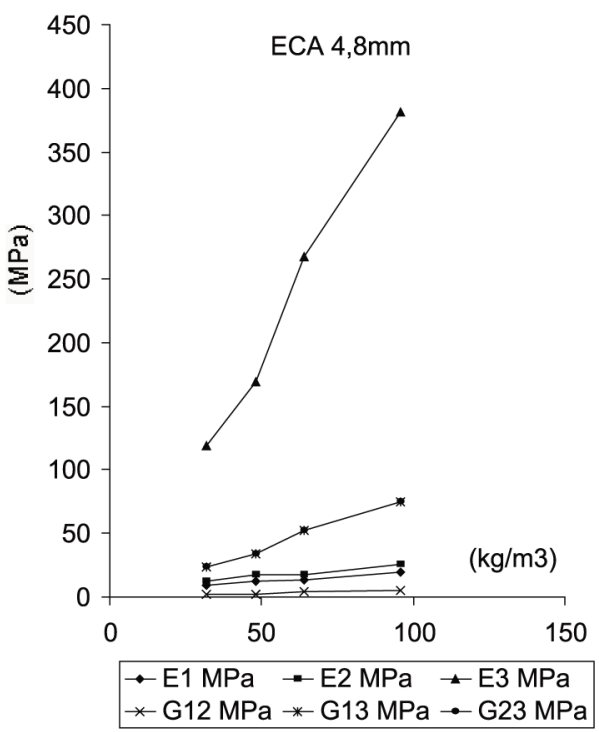

Fig. 8. Évolution des propriétés mécaniques du ECA 4,8 en fonction de sa masse volumique.

d'un essai mécanique de cisaillement [12] ou de compression [19] hors plan, dès que la force atteint son maximum, une chute plus ou moins brutale de celle-ci est observée. Cette chute correspond à des plissements des parois. Ces matériaux sont connus pour avoir un comportement mécanique élastique linéaire fragile jusqu'à leur charge critique. Dans cette étude, nous considérerons que 
Tableau 4. Comparaisons entre les caractéristiques éléments finis (EF) et constructeur (EC). Modules de cisaillement minimum (Min) et typiques (Typ) des nids d'abeilles ECA-R 6,4 de densité 48, 56 et $64 \mathrm{~kg} . \mathrm{m}^{-3}$.

\begin{tabular}{cccccccccccccc}
\hline \multirow{2}{*}{ ECA -R } & \multicolumn{3}{c}{$6,4-48(51)$} & \multicolumn{3}{c}{$6,4-56(51)$} & \multicolumn{4}{c}{$6,4-64(51)$} \\
& \multicolumn{2}{c}{ Min } & \multicolumn{2}{c}{ Typ } & \multicolumn{2}{c}{ Min } & \multicolumn{2}{c}{ Typ } & \multicolumn{2}{c}{ Min } & \multicolumn{2}{c}{ Typ } \\
\hline MPa & EF & EC & EF & EC & EF & EC & EF & EC & EF & EC & EF & EC \\
\hline$G_{13}$ W & 28,6 & 33 & 38,7 & 42 & 32,5 & 33 & 42,4 & 42 & 36,9 & 40 & 49,0 & 56 \\
$G_{23}$ L & 17,7 & 15 & 24,0 & 22 & 20,2 & 18 & 26,3 & 24 & 22,9 & 21 & 30,4 & 26 \\
\hline
\end{tabular}

Tableau 5. Propriétés homogénéisées minimum (Min) et typiques (Typ) des nids d'abeilles ECA 4,8 de densité 32 et $48 \mathrm{~kg} \cdot \mathrm{m}^{-3}$, épaisseur de clinquant de (51) $\mu \mathrm{m}$ et ECA 4,8 de $64 \mathrm{~kg} \cdot \mathrm{m}^{-3}$ et $(76) \mu \mathrm{m}$.

\begin{tabular}{|c|c|c|c|c|c|c|}
\hline $\begin{array}{l}\text { ECA } \\
\mathrm{MPa}\end{array}$ & $\begin{array}{c}\text { Min } \\
4,8-32 \\
(51)\end{array}$ & $\begin{array}{c}\text { Typ } \\
4,8-32 \\
(51)\end{array}$ & $\begin{array}{c}\text { Min } \\
4,8-48 \\
(51)\end{array}$ & $\begin{array}{c}\text { Typ } \\
4,8-48 \\
(51)\end{array}$ & $\begin{array}{c}\text { Min } \\
4,8-64 \\
(76)\end{array}$ & $\begin{array}{c}\text { Typ } \\
4,8-64 \\
(76)\end{array}$ \\
\hline$v_{12}$ & \multicolumn{2}{|c|}{0,7807} & \multicolumn{2}{|c|}{0,7807} & \multicolumn{2}{|c|}{0,8457} \\
\hline$v_{13}$ & \multicolumn{2}{|c|}{0,0413} & \multicolumn{2}{|c|}{0,0413} & \multicolumn{2}{|c|}{0,0202} \\
\hline$v_{23}$ & \multicolumn{2}{|c|}{0,0239} & \multicolumn{2}{|c|}{0,0239} & \multicolumn{2}{|c|}{0,0266} \\
\hline$E_{1}$ & 8,8 & 11,9 & 12,5 & 15,3 & 13,5 & 17,4 \\
\hline$E_{2}$ & 12,0 & 16,3 & 17,0 & 20,9 & 17,8 & 23,0 \\
\hline$E_{3}$ & 118,6 & 161,6 & 169,1 & 207,0 & 267,3 & 345,4 \\
\hline$G_{12}$ & 1,7 & 2,3 & 2,4 & 2,9 & 3,9 & 5,1 \\
\hline$G_{13} \mathrm{~W}$ & 15,5 & 21,1 & 22,1 & 27,0 & 33,9 & 43,8 \\
\hline$G_{23} \mathrm{~L}$ & 23,8 & 32,4 & 33,9 & 41,5 & 52,1 & 67,3 \\
\hline
\end{tabular}

cette charge correspond à leur limite d'utilisation. Les déformations critiques sont déterminées pour l'ensemble des cas de chargements. L'étude des modes de flambement élastiques linéaires d'Euler (11) permet de déterminer les déformations critiques (12) :

$$
\left[K+\lambda^{2} K_{\mathrm{s}}\right] \vec{X}_{\lambda}=\overrightarrow{0}
$$

où $K$ est la raideur du VER, $K_{\mathrm{s}}$ représente la raideur géométrique due à la précontrainte s. $\lambda^{2}$ est le coefficient multiplicateur du chargement. $\vec{X}_{\lambda}$ est le mode propre de flambement associé à $\lambda^{2}$. Les déformations critiques sont déterminées par la relation (20)

$$
E_{J}^{\mathrm{c}}=\lambda^{2} E_{J}
$$

Dans le cas d'un matériau dont le comportement homogénéisé est orthotrope nous proposons de déterminer les six contraintes critiques élémentaires associées aux déformations critiques $E_{J}^{\mathrm{c}}$ en appliquant la loi de comportement homogénéisée (13)

$$
\hat{\sigma}_{I}^{\mathrm{c}}=\hat{C}_{I J} \hat{E}_{J}^{\mathrm{c}}
$$

Les contraintes ultimes du matériau ECA-R 4,8 29 (51) sont présentées dans le tableau 9 (Figs. 10 et 11). D'un point de vue physique, la couche de résine casse lors du premier plissement et la résistance de la cellule chute alors de façon très significative. Expérimentalement, le plateau sur la courbe représentant l'effort global en fonction du déplacement correspond à une densification du nid d'abeilles dû à l'écrasement des parois. D'un point de vue industriel, les zones écrasées

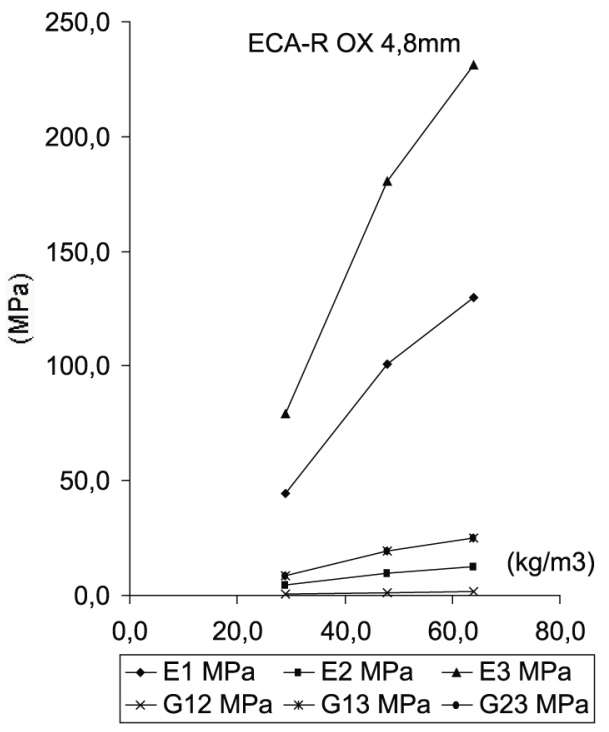

Fig. 9. Évolution des propriétés mécaniques du ECA-R 4,8 en fonction de sa masse volumique.

du Nomex ${ }^{\circledR}$ conduisent à des flambements des peaux du sandwich qui dans certain cas peuvent entraîner la perte du navire. En conséquence, pour ces âmes dont le comportement élémentaire du papier enduit de résine est élastique fragile, des simulations éléments finis des modes de flambement du VER permettent de déterminer correctement les contraintes à rupture de l'âme homogénéisée. Ces contraintes à rupture du matériau orthotrope équivalent sont proches des résultats des essais fournis par le fabricant. Les contraintes ultimes peuvent être utilisées avec un critère de rupture de Hill, cependant cette démarche doit encore être validée expérimentalement. Ces premiers résultats sont très encourageants et montre la pertinence de la modélisation retenue dans cette étude. L'étude du premier mode de flambement statique des VER fournit des résultats dignes de confiance. Cependant, il convient de rappeler que de nombreux paramètres influent sur le flambement. Les plus connus sont le comportement du matériau, les défauts géométriques, les charges suiveuses ou encore, les conditions limites. Il est important de parfaitement maîtriser dans la simulation éléments finis, l'effet de chaque paramètre si l'on veut prédire de façon fiable le flambement qui conduit dans notre cas à la rupture du nid d'abeilles. 
Tableau 6. Comparaisons entre les caractéristiques éléments finis (EF) et constructeur (EC). Modules de cisaillement minimum (Min) et typiques (Typ) des nids d'abeilles ECA 4,8 de densité 32, 48 et 64 kg.m³.

\begin{tabular}{cccccccccccccc}
\hline \multirow{2}{*}{ ECA } & \multicolumn{3}{c}{$\begin{array}{c}4,8-32 \\
(51)\end{array}$} & \multicolumn{3}{c}{$4,8-48(51)$} & \multicolumn{3}{c}{$4,8-64(76)$} \\
& \multicolumn{2}{c}{ Min } & \multicolumn{2}{c}{ Typ } & \multicolumn{2}{c}{ Min } & \multicolumn{2}{c}{ Typ } & \multicolumn{2}{c}{ Min } & \multicolumn{2}{c}{ Typ } \\
\hline MPa & EF & EC & EF & EC & EF & EC & EF & EC & EF & EC & EF & EC \\
\hline$G_{13}$ W & 15,5 & 16 & 21,1 & 22 & 22,1 & 22 & 27,0 & 28 & 33,9 & 34 & 43,8 & 46 \\
$G_{23}$ L & 23,8 & 23 & 32,4 & 31 & 33,9 & 34 & 41,5 & 40 & 52,1 & 52 & 67,3 & 64 \\
\hline
\end{tabular}

Tableau 7. Propriétés homogénéisées minimum (Min) et typiques (Typ) des nids d'abeilles ECA 4,8-96 et ECA 6,4 de densité, 50 et $64 \mathrm{~kg} \cdot \mathrm{m}^{-3}$, épaisseur de clinquant de $(76) \mu \mathrm{m}$.

\begin{tabular}{lcccccc}
\hline ECA & Min & Typ & Min & Typ & Min & Typ \\
MPa & $\begin{array}{c}4,8-96 \\
(76)\end{array}$ & $\begin{array}{c}4,8-96 \\
(76)\end{array}$ & $\begin{array}{c}6,4-50 \\
(76)\end{array}$ & $\begin{array}{c}6,4-50 \\
(76)\end{array}$ & $\begin{array}{c}6,4-64 \\
(76)\end{array}$ & $\begin{array}{c}6,4-64 \\
(76)\end{array}$ \\
\hline$v_{12}$ & \multicolumn{2}{c}{0,8457} & \multicolumn{2}{c}{0,8199} & 0,8199 \\
$v_{13}$ & 0,0202 & \multicolumn{2}{c}{0,0257} & 0,0257 \\
$v_{23}$ & 0,0266 & \multicolumn{2}{c}{0,0338} & 0,0338 \\
\hline$E_{1}$ & 19,3 & 22,0 & 10,0 & 14,4 & 17,0 & 21,0 \\
$E_{2}$ & 25,4 & 29,1 & 13,2 & 18,9 & 22,4 & 27,7 \\
$E_{3}$ & 381,3 & 436,1 & 156,1 & 223,7 & 265,1 & 327,6 \\
\hline$G_{12}$ & 5,6 & 6,4 & 2,8 & 4,0 & 4,7 & 5,8 \\
$G_{13} \mathrm{~W}$ & 48,4 & 55,3 & 19,7 & 28,2 & 33,5 & 41,4 \\
$G_{23} \mathrm{~L}$ & 74,3 & 85,0 & 30,4 & 43,6 & 51,7 & 63,9 \\
\hline
\end{tabular}

\section{Modélisation du pontage}

Sur un voilier multicoque de 60 pieds, la structure est construite en matériaux composites sandwichs et elle subit des sollicitations de type flexion ou torsion. Une structure sandwich est composée d'une âme et de deux peaux en carbone. L'assemblage est réalisé par collage à l'aide de résine compatible avec les matériaux en présence. Les âmes les plus utilisées sont de type nid d'abeilles Nomex ${ }^{\circledR}$. Les peaux sont généralement constituées de structures stratifiées. Ces structures ont une grande rigidité en flexion et torsion. L'âme de la structure sandwich résiste principalement aux contraintes de cisaillement, de traction et de compression hors plan, les peaux inférieures et supérieures supportent quant à elles les efforts dans leur plan.

Les peaux résistent principalement aux contraintes de traction et de compression générées par les moments de flexion. Elles doivent également résister à des flambements locaux qui peuvent être générés par des impacts hydrodynamiques. L'âme doit résister aux contraintes de cisaillement générées par le glissement des deux peaux sous l'action des chargements. Une rupture en cisaillement de l'âme générera un mouvement indépendant des peaux qui conduira à terme à des flambements des peaux et à leur destruction. Dans certain cas, le renforcement en cisaillement hors plan du panneau sandwich est réalisé par pontage local des peaux. Le milieu de la zone pontée est alors généralement renforcé par une âme en mousse (Fig. 12). Ces mousses sont très élastiques avec un faible module de cisaillement et ne se rompent généralement pas de façon catastrophique sous des sollicitations de cisaillement. Il convient de noter

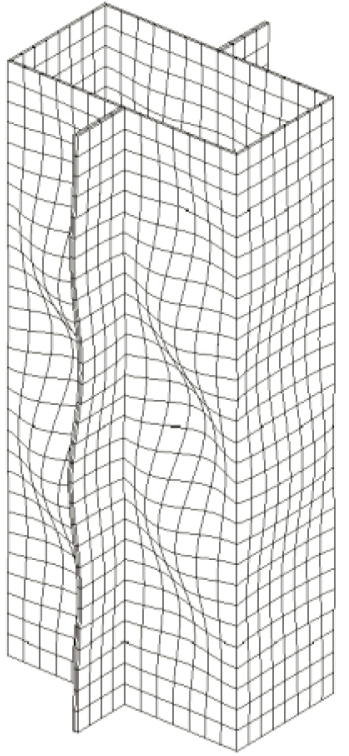

Fig. 10. Déformée de flambement du VER sur-expansé sous l'action d'un chargement de cisaillement hors plan $E_{13}$.

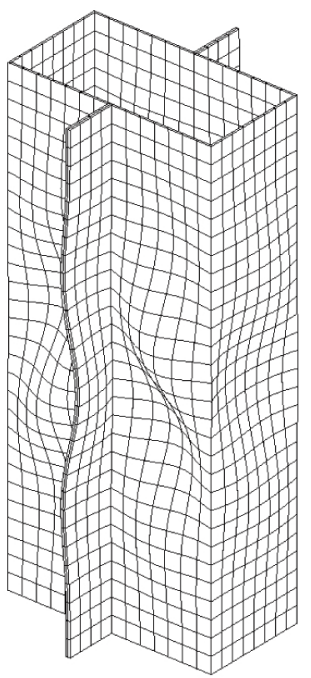

Fig. 11. Déformée de flambement du VER sur-expansé sous l'action d'un chargement de cisaillement hors plan $E_{23}$.

cependant que la mousse est beaucoup plus dense que les nids d'abeilles et son manque de stabilité en température limite son utilisation dans le domaine de la compétition car les voiliers naviguent également dans des régions très chaudes. 
Tableau 8. Comparaisons entre les caractéristiques éléments finis (EF) et constructeur (EC). Modules de cisaillement minimum (Min) et typiques (Typ) des nids d'abeilles ECA 4,8-96 et ECA 6,4 de densité, 50 et 64 kg.m³.

\begin{tabular}{cccccccccccccc}
\hline \multirow{2}{*}{ ECA } & \multicolumn{3}{c}{$4,8-96(76)$} & \multicolumn{4}{c}{$6,4-50(76)$} & \multicolumn{4}{c}{$6,4-64(76)$} \\
& \multicolumn{2}{c}{ Min } & \multicolumn{2}{c}{ Typ } & \multicolumn{2}{c}{ Min } & \multicolumn{2}{c}{ Typ } & \multicolumn{2}{c}{ Min } & Typ \\
\hline $\mathrm{MPa}$ & EF & EC & EF & EC & EF & EC & EF & EC & EF & EC & EF & EC \\
\hline$G_{13}$ W & 48,4 & 46 & 55,3 & 56 & 19,7 & 20 & 28,2 & 28 & 33,5 & 32 & 41,4 & 40 \\
$G_{23} \mathrm{~L}$ & 74,3 & 78 & 85,0 & 84 & 30,4 & 30 & 43,6 & 40 & 51,7 & 56 & 63,9 & 64 \\
\hline
\end{tabular}

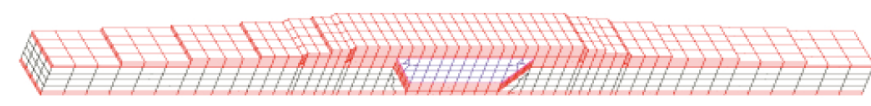

Fig. 12. Patch de réparation en cisaillement par pontage.

Tableau 9. Comparaisons entre les caractéristiques éléments finis (EF) et les essais mécaniques (Essais).

\begin{tabular}{ccccc}
\hline ECA-R & Min & Min & Typ & Typ \\
& EF & Essais & EF & Essais \\
MPa & $4,8-29$ & $4,8-29$ & $4,8-29$ & $4,8-29$ \\
& $(51)$ & $(51)$ & $(51)$ & $(51)$ \\
\hline$\sigma_{11}^{\mathrm{C}}$ & 0,185 & - & 0,31 & - \\
$\sigma_{22}^{\mathrm{C}}$ & 0,282 & - & 0,48 & - \\
$\sigma_{33}^{\mathrm{C}}$ & 0,39 & 0,6 & 0,62 & 0,85 \\
\hline$\sigma_{12}^{\mathrm{C}}$ & 0,72 & - & 1,2 & - \\
$\sigma_{13}^{\mathrm{C}}$ & 0,33 & 0,32 & 0,55 & 0,44 \\
$\sigma_{23}^{\mathrm{C}}$ & 0,25 & 0,31 & 0,415 & 0,42 \\
\hline
\end{tabular}

L'influence du pontage des peaux sur le comportement de membrane et de flexion et torsion du sandwich peut être évalué en utilisant l'homogénéisation du milieu tridimensionnel (Fig. 12). Les caractéristiques mécaniques de la plaque équivalente $[21,22]$ sont déterminées par homogénéisation de la structure tridimentionnelle hétérogène afin de vérifier les couplages mécaniques créés par le pontage et la reprise en scarf de plusieurs peaux. Le comportement plan d'une plaque sandwich correspond à une cinématique de Love-Kirchhoff [23]. Ce comportement de la plaque est évalué par la résolution de six problèmes dont les chargements sont des déformations homogènes imposées sur le contour du VER du pontage. Dans la base globale $\vec{x}, \vec{y}, \vec{z}$. On pose la relation de comportement des plaques sous la forme suivante [17]

$$
\left[\begin{array}{l}
{[\hat{\bar{N}}]} \\
{[\hat{\bar{M}}]}
\end{array}\right]=\left[\begin{array}{l}
{[\hat{\bar{A}}]} \\
{[\hat{\bar{B}}]} \\
{[\hat{\bar{B}}]}
\end{array}\right]\left[\begin{array}{l}
{[\hat{\bar{D}}]} \\
{[\hat{\bar{\chi}}]}
\end{array}\right]
$$

où $\gamma$ représente les déformations de membrane et $\chi$ représente les courbures. La quantité $z \hat{\chi}$ représente les déformations de flexion ou de torsion. Les expressions des efforts généralisés en fonction des contraintes dans chacune des couches s'écrivent :

$$
\hat{\bar{N}}^{T}=\left[N_{x x}, N_{y y}, \sqrt{2} N_{x y}, M_{x x}, M_{y y}, \sqrt{2} M_{x y}\right]
$$

Si une structure est stratifiée de façon quelconque ou n'est pas équilibrée, il existe un couplage entre le comportement membranaire et le comportement de flexion et de torsion. Ce couplage est dû à la matrice $[\hat{\bar{B}}]$. La structure sandwich de base est constituée d'une âme en Nomex ${ }^{\circledR}$ et de deux peaux en tissu carbone à deux directions de renforcement. Elle possède un comportement de plaque homogénéisée avec un couplage faible qui s'écrit sous la forme suivante

$$
\begin{aligned}
& {[\hat{\bar{A}}]=\left[\begin{array}{ccc}
5,355 \times 10^{5} & 0 & 0 \\
0 & 2,398 \times 10^{7} & 0 \\
0 & 0 & 1,765 \times 10^{7}
\end{array}\right],} \\
& {[\hat{\bar{B}}]=\left[\begin{array}{ccc}
0 & 0 & 0 \\
1,5556 \times 10^{1} & 0 & 0 \\
0 & 0 & 0
\end{array}\right]} \\
& {[\hat{\bar{D}}]=\left[\begin{array}{ccc}
1,534 \times 10^{6} & 0 & 0 \\
0 & 9,761 \times 10^{4} & 0 \\
0 & 0 & 7,53 \times 10^{3}
\end{array}\right]}
\end{aligned}
$$

La structure sandwich renforcée par le pontage et une âme en mousse possède un comportement de plaque homogénéisée plus fortement couplé

$$
\begin{aligned}
& {[\hat{\bar{A}}]=\left[\begin{array}{ccc}
8,299 \times 10^{5} & -2,57 \times 10^{6} & 0 \\
-2,57 \times 10^{6} & 5,225 \times 10^{7} & 0 \\
0 & 0 & 3,297 \times 10^{7}
\end{array}\right],} \\
& {[\hat{\bar{B}}]=\left[\begin{array}{ccc}
3,514 \times 10^{1} & 0 & 0 \\
-7,062 \times 10^{2} & 0 & 0 \\
0 & 0 & 0
\end{array}\right]} \\
& {[\hat{\bar{D}}]=\left[\begin{array}{ccc}
5,413 \times 10^{6} & 0 & 0 \\
0 & 5,347 \times 10^{3} & 0 \\
0 & 0 & 1,079 \times 10^{4}
\end{array}\right]}
\end{aligned}
$$

La détermination précise du comportement mécanique d'une réparation ne peut être réalisée que par une approche fine du comportement de chacun des constituants (tissus, âmes). La résistance au cisaillement hors plan d'une plaque sandwich est fortement accrue par la présence d'un pontage des peaux, cependant, cette solution engendre des couplages membrane flexion.

\section{Conclusions}

De nombreux travaux scientifiques concernant le comportement des nids d'abeilles ont été réalisés afin de simuler le comportement mécanique homogène équivalent de ces âmes. L'étude montre que l'approche retenue permet également de retrouver par simulation éléments finis les 
contraintes ultimes de cisaillement fournies par les fabricants. La démarche retenue permet de calculer l'ensemble des contraintes ultimes et permet de créer une enveloppe de rupture des âmes. Il convient de noter que des études expérimentales complémentaires devraient être réalisés afin de valider entièrement cette démarche. Les coefficients et limites de rupture des âmes en nids d'abeilles permettent de nourrir nos modèles éléments finis tridimensionnels dédiés à l'étude du renforcement des structures sandwichs utilisées sur les trimarans océaniques de 60 pieds.

\section{Références}

[1] L. Gornet, G. Marckmann, Analyse par éléments finis de réparation de trimarans, plans Marc Lombard, rapport interne LMM 2002

[2] C. Elbeze, D. Bourgeois, Martin-Raget, La Route du Rhum 2002, Larivière, 2002

[3] F. Cammas, A. Gautier, B. De Broc, M. Lombard, Le Rhum en questions, Voiles et voiliers, France Métro, 383 (2003) 74-91

[4] P. Suquet, Plasticité et homogénéisation, Thèse de doctorat d'état, Université Pierre et Marie Curie, Paris 6, 1982

[5] O. Debordes, Homogenization computations in elastic or plastic range; applications to unidirectional composites and perforated sheets, 4th Int. Symp. Innovative Num. Method in Engng, Computational Mechanics Publications, Springer-Verlag, Atlanta, 1982, pp. 453-458

[6] F. Lene, Contribution à l'étude des matériaux composites et de leurs endommagements, Thèse de doctorat, Université Paris VI, 1984

[7] P. Verpeaux, T. Charras, A. Millard, Castem 2000 : Une approche moderne du calcul des structures, J.M. Fouet, P. Ladevèze, R. Ohayon (ed.), 2 1988, pp. 227-261

[8] Nids d'abeilles Nomex ${ }^{\circledR}$, http://www . euro-composites.com

[9] S. Kelsey, R.A. Gellatly, B.W. Clark, The shear modulus of foil honeycomb core, Aircraft Engng., 1958, pp. 294-302
[10] L.J. Gibson, M.F. Ashby, Cellular solids structures and properties, Pergamon Press, Oxford, 1988

[11] L.J. Gibson, M.F. Ashby, G.S. Shajer, C.I. Robertson, The mechanics of two-dimensional cellular materials, Proc. R. Soc. Lond. A 382 (1982) 25-42

[12] J.F. Shang, M.F. Ashby, The Out-of-Plane properties of honeycombs, Int. J. Mech. Sci. 34 (1992) 475-489

[13] M. Grediac, A finite element study of the transverse shear in honeycomb cores, Int. J. Solids Structures 30 (1993) $1777-1788$

[14] J. Hohe, W. Becker, A refined analysis of the effective elasticity tensor for general cellular sandwich cores, Int. J. Solids Structures 38 (2001) 3689-3717

[15] J. Hohe, W. Becker, An energetic homogenisation procedure for the elastic properties of general cellular sandwich cores, Composit Part B 32 (2001) 185-197

[16] L. Gornet, J. Royer, S. Sablin, Prévision de la dégradation de composites carbone époxy stratifiés : application aux voiliers multicoques, JNC 12, Cachan France, $12^{\mathrm{e}}$ journées nationales sur les composites, 2000, pp. $51-59$

[17] L. Gornet, Comportement mécanique et analyse des structures en matériaux composites, cours École centrale Nantes, 2001

[18] C.T. Herakovich, Mechanics of fibrous composite, John Wiley, 1998

[19] Y. Aminanda, B. Castanie, J.J. Barrau, P. Thevenet, Étude expérimentale et modélisation de l'indention des structures sandwichs à âme nid d'abeilles, JNC13, Y. Rémond, J. Lamon (ed.), 2003, pp. 461-470

[20] A. Combescure, Étude de la stabilité non-linéaire géométrique et non-linéaire matériau des coques minces, Habilitation à Diriger les Recherches de UCB Lyon, 1995

[21] S. Bourgois, Modélisation numérique des panneaux structuraux légers, Thèse de doctorat, Université de Méditerranée (Aix-Marseille II), 1997

[22] N. Buannic, P. Cartraud, T. Quesnel, Homogenization of corrugated core sandwich panels, Composite Structures 59(3) (2003) 299-312

[23] J.N. Reddy, Mechanics of Laminated Composite Plates: Theory and Analysis, CRC Press, 1997 